\title{
Summation Calculations for Reactor Antineutrino Spectra, Decay Heat and Delayed Neutron Fractions Involving New TAGS Data and Evaluated Databases
}

M. Estienne ${ }^{1, *}, M$. Fallot ${ }^{1,}, L$. Giot ${ }^{1}$, , V. Guadilla-Gomez ${ }^{1}$, L. Le Meur ${ }^{1}, A$. Porta ${ }^{1}, A$. Algora $^{2,13}, J$. -L. Taìn ${ }^{2}, J$. A. Briz ${ }^{1}, J$. Agramunt ${ }^{2}, J$. Äystö ${ }^{4}, S$. Cormon ${ }^{1}, A$. Cucoanes ${ }^{1}, T$. Eronen $^{7}$, L. M. Fraile ${ }^{15}, E$. Ganogliu ${ }^{17}, W$. Gelletly ${ }^{2,3}, D$. Gorelov ${ }^{7}, J$. Hakala ${ }^{7}, A$. Jokinen ${ }^{7}$,

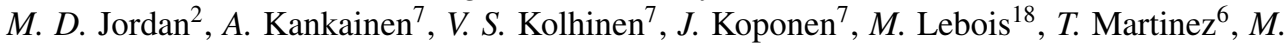
Monserrate $^{2}$, A. Montaner-Pizá ${ }^{2}, I$. Moore ${ }^{7}, E$. Nácher $^{19}$, S. E. A. Orrigo ${ }^{2}, H$. Penttilä ${ }^{7}, I$. Pohjalainen $^{7}, J$. Reinikainen ${ }^{7}, M$. Reponen ${ }^{7,10}, S$. Rinta-Antila ${ }^{7}, K$. Rytkönen, B. Rubio ${ }^{2}, T$.

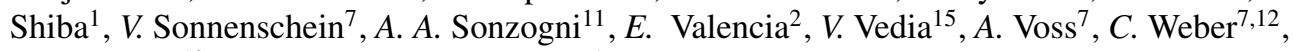
$J$. N. Wilson ${ }^{18}$, and $A$. $-A$. Zakari-Issoufou ${ }^{1}$

${ }^{1}$ Subatech, CNRS/IN2P3, 4 Rue Alfred Kastler, 44307 Nantes, France

${ }^{2}$ IFIC (CSIC-Univ. Valencia), Valencia, Spain

${ }^{3}$ Department of Physics, University of Surrey, Guildford, GUZ7XH, UK

${ }^{4}$ Helsinki Institute of Physics, FI-00014 University of Helsinki, Finland

${ }^{5}$ Universitat Politécnica de Catalunya (UPC), 08034, Barcelona, Spain

${ }^{6}$ CIEMAT, Madrid, Spain

${ }^{7}$ Department of Physics, P.O. Box 35, FI-40014 University of Jyväskylä, Finland

${ }^{8}$ Argonne National Laboratory, Argonne, USA

${ }^{9}$ National Physical Laboratory, Teddington, Middlesex, TW11 0LW, UK

${ }^{10}$ RIKEN, 2-1 Hirosawa, Wako, Saitama 351-0198, Japan

${ }^{11}$ National Nuclear Data Center, Brookhaven National Laboratory, Upton NY 11973-5000, USA

${ }^{12}$ Faculty of Physics, Ludwig-Maximilians University Munich, Am Coulombwall 1, D-85748 Garching, Germany

${ }^{13}$ MTA ATOMKI, Hungary

${ }^{14}$ Petersburg Nuclear Physics Institute, RU-188300 Gatchina, Russia

${ }^{15}$ Universidad Complutense, Grupo de Física Nuclear, CEI Moncloa, E-28040, Madrid, Spain

${ }^{16}$ United Kingdom Atomic Energy Authority, Culham Science Centre, Abingdon, OX14 3DB, UK

${ }^{17}$ Department of Physics, Istanbul University, 34134, Istanbul, Turkey

${ }^{18}$ Institut de Physique Nucléaire d'Orsay, CNRS/IN2P3, F-91406 Orsay, France

${ }^{19}$ Instituto de Estructura de la Materia, CSIC, E-28006, Madrid, Spain

Abstract. Three observables of interest for present and future reactors depend on the $\beta$ decay properties of the fission products: antineutrinos from reactors, the reactor decay heat and delayed neutron emission. In these proceedings, we present new results from summation calculations of the first two quantities quoted above, performed with evolved independent yields coupled with fission product decay data, from various nuclear data bases or models. New TAGS results from the latest experiment of the TAGS collaboration at the JYFL facility of Jyväskylä will be displayed as well as their impact on the antineutrino spectra and the decay heat associated to fission pulses of the main actinides.

*e-mail: magali.estienne@subatech.in2p3.fr 


\section{Introduction}

The study of $\beta$ decay is useful to better constrain different domains of physics achievable close to reactors. Its implications for present and future reactors concern the physics of antineutrinos and their applications, the decay heat (DH) understanding and control and the access to the delayed neutron fractions. They are essential respectively for fundamental physics (neutrino oscillation, mass hierarchy and reactor anomaly), reactor monitoring and non proliferation, reactor safety and economy as concerns DH and the operation and control of the chain reactions in reactors. Indeed, in a PWR the thermal power is mainly induced by the fission process undergone by the four isotopes ${ }^{235} \mathrm{U},{ }^{239} \mathrm{Pu},{ }^{241} \mathrm{Pu}$ and ${ }^{238} \mathrm{U}$. Fission products (FP) are released in such process, at the origin of the $\beta$ decays as they are neutron rich nuclei. In such radioactive processes, a neutron from the parent nucleus converts into a proton in the daughter nucleus and emits an electron and an electron antineutrino. Gamma-rays are also emitted in the de-excitation of the produced daughter nucleus. In the specific case of the production of the daughter in an excited state above the neutron energy threshold, a neutron can be emitted before the de-excitation of a second daughter nucleus. This is known as a $\beta-n$ decay associated to the emission of a delayed neutron. Whether the $\gamma$ and $\beta$ particles, the $\beta$ delayed neutrons or the neutrinos, their independent measurement allows to assess $\beta$ decay physics properties and applications. In these proceedings, we will essentially focus on the physics that can be addressed via the $\gamma$ channel.

In the next section, experimental issues associated to $\gamma$ measurement will be introduced before to present the Total Absorption Gamma-ray Spectroscopy (TAGS) technique used to overcome experimental biases. Two of the 3 introduced $\beta$-decay applications will be commented below: antineutrinos from reactors in Sect. 3 and decay heat in Sect. 4. Both aspects will be discussed in the context of the summation calculations that have been developed by the Nantes group and that will be introduced and commented in Sect. 3.2 and 4.2. The summation method is also a useful tool to give predictions for delayed neutron fractions but these aspects are not presented in the present paper. They have been the focus of a Coordinated Research Program of the IAEA attended by our group dedicated to the establishment of a new $\beta$-delayed neutron nuclear database (DB) which outcome is the object of a publication in preparation [1]. A general conclusion will be given in Sect. 5 .

\section{Pandemonium Effect and Total Absorption Gamma-ray Spectroscopy (TAGS)}

\subsection{Pandemonium Bias in Nuclear Databases}

A precise measurement of the $\beta$ decay probability, the $\beta$ intensity $I_{\beta}$, is essential for the determination of the $\beta$ strength which characterizes the structure properties of a nucleus. It is achievable with a clean determination of the energy levels of the daughter nucleus via the $\gamma$-cascade measurement. The experimental knowledge of the endpoints, the Q-value and the Branching Ratios (BR) of a given FP giving access to its $\beta /$ anti- $v$ energy spectra, it becomes possible to calculate a complete $\beta /$ anti- $\nu$ spectra of the 4 main fissible nuclei present in the reactor core of a PWR. The access to the mean $\gamma$ and $\beta$ energies of a given FP also brings a stone to add to the building of the DH determination and enriches as-well nuclear databases.

Before the 1990s, high resolution $\gamma$-ray spectroscopy through the use of germanium detectors was the conventional detection technique used for $\gamma$-ray measurement. It offers excellent energy resolution but an efficiency which strongly decreases at high energy and usually limited acceptance. As direct consequences, weak $\gamma$-ray cascades are likely difficult to detect and the decay scheme of the parent nucleus may be incomplete. There is a danger of 
overlooking the existence of $\beta$-feeding into the high energy nuclear levels of daughter nuclei especially with decay schemes with large Q-values. This phenomenon is called "Pandemonium effect" highlighted by Hardy in 1977 [2]. The $\beta$ decay of the ${ }^{105}$ Mo is a good illustration [3]. A comparison between the calculated antineutrino spectra obtained using the results of a measurement performed with high resolution technique (JEFF 3.1.1) and the TAS one (see Sect. 2.2) which corrects for that effect can be made. A $\simeq 50 \%$ discrepancy is observed in the mean energy of the distributions to be compared with the few percent uncertainty quoted in JEFF 3.1.1 DB. This example highlights the strong potential bias present in nuclear DB for nuclei suffering for Pandemonium effect not only concerning the mean energy values, level energies, etc. but also concerning their associated uncertainties. It might have subsequent consequences while using nuclear DB in $\beta$ decay applications that need careful treatment.

\subsection{The Total Absorption $\gamma$-ray Spectroscopy}

\subsubsection{TAS calorimeter and experimental campaigns}

A solution proposed to solve the above-mentioned issue was to use a calorimeter containing big crystals ideally covering $4 \pi$ : the Total Absorption $\gamma$-ray Spectroscopy (TAGS). The detection philosophy with respect to HPGe is totally different. Instead of detecting the $\gamma$ rays individually, a TAS is used to absorb the full $\gamma$-ray energy released by the $\gamma$ cascades in the $\beta$ decay process. It offers an excellent efficiency but a lower resolution than HPGE (see below). Apart from the detector inefficiency that has to be corrected for, this technique gives directly access to the energy level in the daughter nucleus. The first TAS developed in the 1970s were too small detectors to be enough efficient. The development of a TAGS method efficient and systematic appeared in the 1990s [4] and has been improved since then. TAS are now segmented allowing "multiplicity" measurements and the analysis methodology has been noticeably improved [5].

The European TAS collaboration has performed so far two experimental campaigns of data taking with two detectors built by the Valencia and Surrey groups at the IGISOL facility in Jyväskylä [7]. The first used in 2009, made of $12 B a F_{2}$ crystals offering a detection efficiency of $\gamma$-ray cascade larger than $80 \%$, was coupled with a silicon detector for $\beta$ coincidences to reduce the background contribution. Seven nuclei of interest for both antineutrino and decay heat applications were measured during this campaign [6]. The second one in 2014, DTAS, a 18 -fold segmented $\mathrm{NaI}(\mathrm{Tl})$ spectrometer with rectangular crystals of $15 \times 15 \times 25 \mathrm{~cm}^{3}$, has also an excellent efficiency of $80-90 \%$ to detect a single $\gamma$-ray associated with an individual crystal energy resolution of 7-8\%. It is coupled with a plastic detector for $\beta$ coincidences. This experiment was very successful as 23 nuclei (from the Nantes/Valencia's proposals) of interest for antineutrino and $\mathrm{DH}^{1}$ [8] were measured. Among the 15 nuclei analyzed so far some preliminary results for the ${ }^{99} \mathrm{Y},{ }^{138} \mathrm{I},{ }^{142} \mathrm{Cs}$ [9], ${ }^{100,100 m} \mathrm{Nb}$ and ${ }^{102,102 m} \mathrm{Nb}$ [10] nuclei are presented below.

\subsubsection{Analysis: determination of the $\beta$ intensity $I_{\beta}$}

We show in the top panel of Fig. 1 preliminary results of the $\beta$-gated energy spectrum of gammas for the ${ }^{99} \mathrm{Y}$ nucleus, a priority 1 contributor to the spectrum [8]. In the figure, the locations of the Q-value and the neutron emission threshold are indicated by vertical lines. The $\beta-\gamma$ coincidences guarantee a spectrum free from environmental background, however, all other sources of contamination have to be carefully studied in the analysis. The green

\footnotetext{
${ }^{1}$ The Nantes group is taking part to the TAGS consultant meetings of the IAEA dedicated to the elaboration of the priority list of Pandemonium nuclei that need to be measured.
} 
line represents the contribution of the pile-up signals to the data extracted from a MC study, the blue one the contamination from the daughter ${ }^{99} \mathrm{Zr}$ nucleus determined solving the Bateman equations and the purple line the contamination from $\beta$-delayed neutrons. The red line is the result of the reconstruction of the TAGS data with the feeding found with the Bayes method [11]. It is already in very good agreement with the data shown in black. The resulting preliminary $I_{\beta}$ distribution (without the $\beta$-n contribution) is shown in the bottom panel of Fig. 1 where the feeding is compared with the ENSDF database containing experimental results from high-resolution measurements. The discrepancy between the two feedings highlights how the Pandemonium problem biases high-resolution data and consequently nuclear databases. The shaded areas reflect the envelop of systematic errors obtained in varying the input ingredients entering in the data analysis.
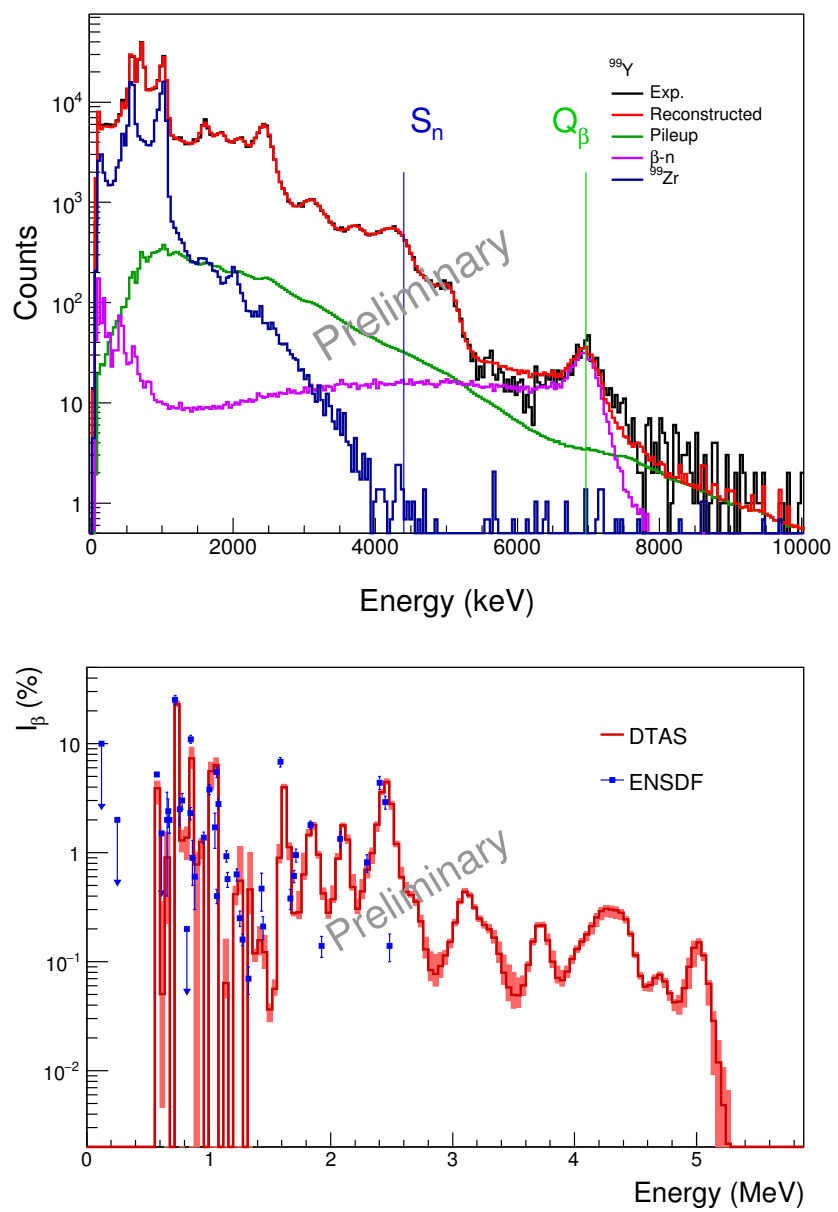

Figure 1. Top: Experimental TAS spectrum of ${ }^{99} \mathrm{Y}$ showing also contaminants (see text) and the reconstruction obtained after the application of the Bayes method (red line). Bottom: Beta intensity of the beta decay of ${ }^{99} \mathrm{Y}$ (see text). 


\section{Reactor Antineutrinos}

\subsection{Physics motivations}

$\beta$ decays of FP are responsible for the huge flux of antineutrinos emitted close to reactors. Taking into account that $\sim 6$ antineutrinos are emitted per fission ${ }^{2}$, a PWR of $1 G W e$ releases a flux of the order of $10^{21} / \mathrm{s}$ antineutrinos. With such a neutrino factory, two domains of study hitting for the first applied physics and for the second fundamental physics become attainable. Measuring a flux and a spectral shape should allow to measure the composition of the reactor core. Indeed, it was proposed in the 1970s (and then demonstrated in the 1980s/90s) to use the discrepancy between antineutrino flux and energies from $\mathrm{U}$ and $\mathrm{Pu}$ isotopes to infer reactor fuel isotopic composition and power. Measuring a flux and a spectral shape would also allow the measurement of an oscillation if it exists. This second domain of study is of the interest of fundamental neutrino physics. Across the years, neutrino experiments have found anomalies in their data, i.e. data which could not be explained by an oscillation scheme with three neutrino flavors. The "reactor anomaly" designates the deficit observed between the detected antineutrino flux by reactor neutrino experiments at less than $100 \mathrm{~m}$ from reactors with respect to a new prediction of this detected flux [12]. The latter new prediction has been obtained through a new conversion of the integral $\beta$ energy spectra $[13,14]$ measured by Schreckenbach et al. [15] into antineutrinos. These changes led to a new normalisation of the converted detected antineutrino flux lying $6 \%$ above the detected flux at short distances from reactors, when accounting for out-of-equilibrium effects and a new neutron lifetime value [12]. Moreover, the comparison between the converted spectra and the measured spectra of Daya Bay (DBay), Double Chooz and Reno, apart from the normalisation which confirmed the reactor anomaly, exhibits a large distorsion of the data with respect to the model between 5 and $7 \mathrm{MeV}$ in antineutrino energy called the "bump" [16-18]. Lastly, DBay recently put into question integral $\beta$ measurement of ${ }^{235} \mathrm{U}$ questioning the degree of trust placed in the conversion method [19].

\subsection{Summation Calculations and Recent Experimental Improvements}

The summation method was proposed simultaneously to the conversion method as an alternative allowing to study the sub-components of the reactor antineutrino energy spectrum. The antineutrino spectrum associated with one of the four fissioning isotopes in a moderated reactor can be computed as the sum of the contributions of all FP using the full information available per nucleus in the NDB as described in [20]. This method is useful for several reasons. Not only it is the only one that can be adapted to the computation of the antineutrino emission associated to various reactor designs, but also it allows for the computation of antineutrino spectra for which no integral $\beta$ spectrum has been measured yet taking into account off-equilibrium effects and allowing the use of different energy binnings of interest for reactor neutrino experimental analyses.

As an illustration, we picked-up various results obtained so far. In 2012 [20] we published predictions for the antineutrino spectra for the 4 main isotopes in a reactor core and a direct comparison to the converted spectra highlighting a 5 to $10 \%$ deficit in the ${ }^{235} \mathrm{U}$ spectrum in agreement with DBay [19]. We also published the main contributors to a standard PWR antineutrino energy spectrum in bins of energy of interest for the bump study [21] which are of first priority for new TAGS measurements. The impact on the antineutrino energy spectrum of the seven nuclei ${ }^{99} \mathrm{Y},{ }^{138} \mathrm{I},{ }^{142} \mathrm{Cs},{ }^{100,100 m} \mathrm{Nb}$ and ${ }^{102,102 m} \mathrm{Nb}$ have been studied with the summation method. Their cumulated contributions is illustrated in Fig. 2 for ${ }^{238} \mathrm{U}$ (left),

\footnotetext{
${ }^{2}$ This is an average value which depends on the fissioning system
} 
${ }^{235} \mathrm{U}$ (middle left), ${ }^{239} \mathrm{Pu}$ (middle right) and ${ }^{241} \mathrm{Pu}$ (right). We observe a maximum negative deviation of $\simeq 4 \%$ in an energy range from 3 to $5 \mathrm{MeV}$ and a positive deviation in the region of the bump, deviations which go in the direction of neutrino experiment measurements.

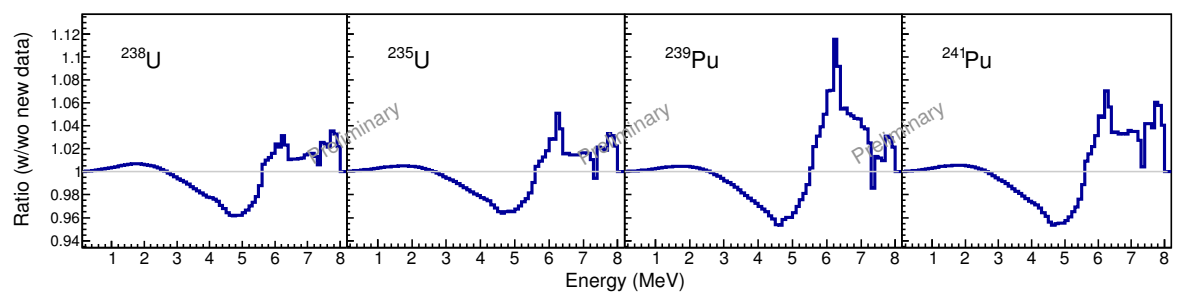

Figure 2. Ratios between 2 spectra built with the dataset described in the text over the same but the TAS data.

\section{Decay Heat}

\subsection{General Motivations for DH Study and Summation Calculations}

Following the shut-down of the chain reaction in a reactor, the nuclear fuel continues to release an energy with time called decay heat. It is essentially made up of radioactive ( $\alpha$, $\beta$ and $\gamma$ radiations) decays of the FP and actinides. Their released energy as a function of the cooling time distributes very differently. In the first 10 s after the reactor stop, the DH is dominated by the delayed fissions, then its main source comes from the FP over 100 years. After this time it is dominated by the actinides decay. On average, it represents a residual power of 6 to $12 \%$ of the nominal power of the reactor just after its shut-down. These numbers are huge and motivate the necessity to have a good knowledge of the DH for both the evaluation of the reactor safety and various economic aspects of nuclear power generation. The DH is estimated through the only method able to be predictive for future reactors: the summation method [22]. The decay heat defined as $f(t)$ can be analytically calculated as the sum over the whole FP and actinides inventoried for specific conditions of reactor operation and subsequent cooling period of their mean $\alpha, \beta, \gamma$ energies times their total decay constant times their fission yield. In the case of fission pulses, the time ranges up to $\sim 10^{6} \mathrm{~s}$ and the fission of pure isotopes is considered.

In the 1970s, important discrepancies were observed between summation calculations which used the information stored in nuclear DB taken worldwide and benchmark experiments (see [23] for a review). With the discovery of the Pandemonium effect as potentially responsible for the observed discrepancies, measured TAS data have been used since the 1990s to solve the problem. Figure 2 in [25] illustrates how the long-standing electromagnetic component of the DH puzzle was partially solved in 2010 following the first TAS campaign in Jyväskylä of the Valencian's group. During this experimental campaign, 7 nuclei of first priority for the decay heat interest were measured and analyzed: the ${ }^{101} \mathrm{Nb},{ }^{105} \mathrm{Mo}$ and the ${ }^{102,104-107} \mathrm{Tc}$. Two summation calculations including or not the 7 TAS nuclei were computed using ENDF/B-VII nuclear DB and compared with the Tobias compilation of integral measurements [23]. Five of the 7 nuclei drastically improved the calculation over the whole cooling time covered by the distribution. This important result motivated the necessity to prolongate the experimental effort and is one of the motivations of the second TAS campaign (Sect. 2.2.1). Indeed the $\gamma$ component of the $\mathrm{DH}$ of the most well known nuclides from 
integral measurements are still not well reproduced by the calculations including different nuclear DB [23].

\subsection{Some Preliminary Results from the $2^{\text {nd }}$ TAS Campaign}

The mean $\beta$ and $\gamma$ energies of the nuclei ${ }^{99} \mathrm{Y},{ }^{138} \mathrm{I},{ }^{142} \mathrm{Cs},{ }^{100,100 m} \mathrm{Nb}$ and ${ }^{102,102 m} \mathrm{Nb}$ have been computed from the $\beta$ intensities experimentally determined since the $2^{\text {nd }}$ TAS campaign. They have been compared with ENDF/B-VII.1 and JEFF 3.3 databases. Firstly, they all present the same systematics: a larger $\bar{E}_{\gamma}$ in TAS compared with the other nuclear DBs and a lower $\bar{E}_{\beta}$ as expected for Pandemonium nuclei. Secondly, their $\Delta \bar{E}$ with the two other DBs are of the order of few hundreds of $\mathrm{keV}$ for both the $\gamma$ and $\beta$ components which is on average twice larger than the uncertainties quoted in nuclear DBs. These two observations illustrate the presence of biases in nuclear DBs which need to be improved with such kind of measurements. We started a collaboration with the CEA Cadarache in order to quantify the impact of the bias in the DB uncertainties on the calculation of the total uncertainties associated with the DH.
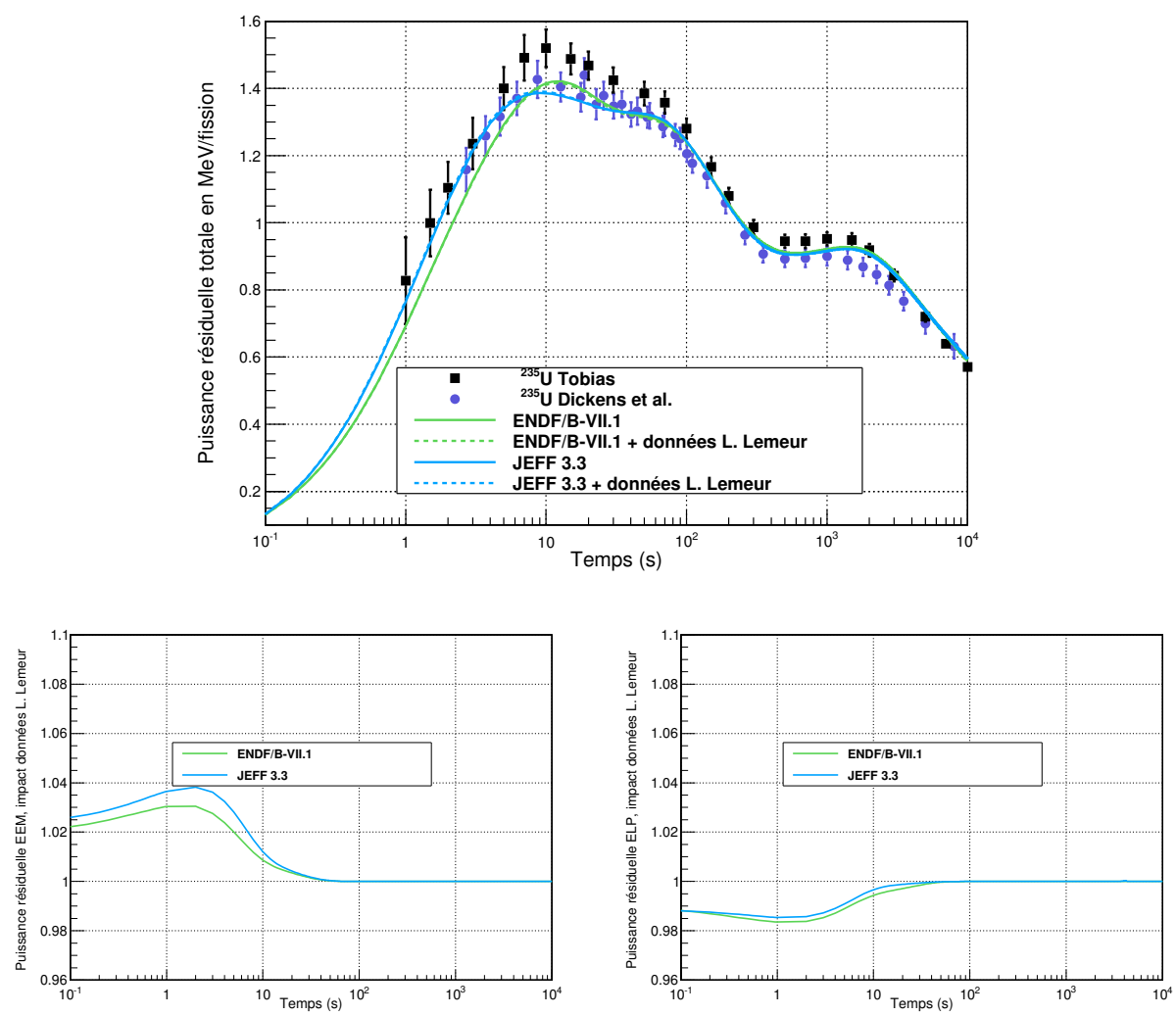

Figure 3. Top: ${ }^{235} \mathrm{U}$ fission pulse total decay heat computed with ENDF/B-VII (green) and JEFF3.3 (blue) and compared to Tobias et. al and Dickens et al [23]. Bottom: impact of the 3 nuclei ${ }^{99} \mathrm{Y},{ }^{138} \mathrm{I}$, ${ }^{142} \mathrm{Cs}$ on the electromagnetic and light particle components of the decay heat respectively, calculated with ENDF/B-VII (green) and JEFF3.3 (blue). 
With the knowledge of the new values of $\bar{E}_{\gamma}$ and $\bar{E}_{\beta}$ it has then been possible to calculate the impact of all these nuclei on the total DH and on the individual electromagnetic (EEM) and light particle (ELP) components. For that purpose, some summation calculations have been performed using Serpent 2. Serpent is a multi-purpose three-dimensional continuousenergy Monte Carlo particle transport code, developed at the VTT Technical Research Centre of Finland [26]. The decay heat has been computed over $10^{4} \mathrm{~s}$ cooling time. It is illustrated for the case of the three nuclei ${ }^{99} \mathrm{Y},{ }^{138} \mathrm{I},{ }^{142} \mathrm{Cs}$ taken together in Fig. 3. The top panel of the figure shows the absolute total $\mathrm{DH}$ for ${ }^{235} \mathrm{U}$ while the two other panels depict the relative impact of the 3 nuclei on the EEM and ELP components of the DH respectively. The calculations have been performed with two nuclear databases: ENDF/B-VII.1 (green) and JEFF 3.3 (blue). While the impact of the sum of the 3 nuclei is lower than $1 \%$ in both DB on the total DH below $10 s$, we can see that it comes from a compensation of the two EEM and ELP components. The left and right panels show that the nuclei have a larger impact on each component taken individually (1-2\% on ELP and 3-4\% on EEM). Note that the main contributions come from the ${ }^{142} \mathrm{Cs}^{3}(2 \%)$ and the ${ }^{99} \mathrm{Y}(1.5 \%)$ but this is not shown here. We have similar conclusions concerning the ${ }^{239} \mathrm{Pu}$. Concerning niobiums, the EEM and ELP components which are also at the level of the percent for both ${ }^{235} \mathrm{U}$ and ${ }^{239} \mathrm{Pu}$ in both DBs compensate for the total $\mathrm{DH}$.

\section{Conclusion}

In conclusion, we have presented in this paper the motivations for new measurements of $\beta$ decay properties of fission products, as key ingredients in the estimate of the reactor decay heat, reactor antineutrinos and $\beta$-delayed neutron fractions. The Total Absorption Gammaray Spectroscopy technique allows to avoid the Pandemonium effect which affects some of the data of evaluated nuclear databases. We have presented new results of a TAGS campaign performed at the IGISOL facility of Jyväskylä, Finland. Their impact on decay heat from fission pulses and on individual antineutrino spectra was assessed and found to be significant.

\section{References}

[1] P. Dimitriou et al., submitted to Nuclear Data Sheet, (dec 2018)

[2] J. C. Hardy, et al., Phys. Lett. B 71, 307 (1977)

[3] M. Estienne, et al., Nucl. Data Sheets 120, 149 (2014)

[4] R. C. Greenwood et al., Nucl. Instrum. Meth. A 314, 514 (1992) and references therein

[5] D. Cano-Ott et al., Nucl. Instrum. Methods Phys. Res., Sect. A430, 488 (1999); 430, 333 (1999). J. L. Tain and D. Cano-Ott, Nucl. Instrum. Methods Phys. Res., Sect. A571, 728 (2007); 571, 719 (2007)

[6] V. Guadilla et al. Characterization and performance of the DTAS detector, Nucl. Instr. Meth. A vol. 910 (2018)

[7] T. Eronen et al., Eur. Phys. J. A 48, 46 (2012)

[8] P. Dimitriou and A. L. Nichols, IAEA report INDC(NDS)-0676, Feb. 2015, IAEA, Vienna, Austria

[9] L. Le Meur, PhD thesis Nantes University, oct. 2018

[10] V. Guadilla-Gomez, PhD thesis Valencia University, sept. 2017

[11] J.L. Taìn et al., Nucl. Instr. Methods Phys. Res. A 571, 728 (2012)

[12] G. Mention et al., Phys. Rev. D 83, 073006 (2011)

[13] Th. Mueller et al., Phys. Rev. C 83, 054615 (2011)

\footnotetext{
${ }^{3}$ which was listed as priority 3 in the IAEA list of priority nuclei to measure for DH
} 
[14] P. Huber, Phys. Rev. C 84, 024617 (2011)

[15] K. Schreckenbach et al., Phys. Lett. 160B, 325 (1985) and references therein

[16] Y. Abe et al., Phys. Rev. Lett. 108, 131801 (2012)

[17] F. P. An et al., Phys. Rev. Lett. 108, 171803 (2012)

[18] J. K. Ahn et al., Phys. Rev. Lett. 108, 191802 (2012)

[19] F. P. An et al. (Daya Bay Collaboration), Phys. Rev. Lett. 118, 251801 (2017)

[20] M. Fallot et al., Phys. Rev. Lett. 109, 202504 (2012)

[21] A.-A. Zakari-Issoufou et al., Phys. Rev. Lett. 115, 102503 (2015)

[22] T. Yoshida et al., OECD/NEA Working Party for Interna- tional Evaluation Cooperation, 1425, 25 (2007). Nuclear Science NEA/WPEC-25 (2007)

[23] M. Fleming and J.-C. Sublet, report 2015 Fispact II, CCFE-R(15) 28 and references therein

[24] K. Takahashi, M. Yamada, T. Kondoh, At. Data Nucl. Data Tables 12 (1), 101-142 (1973). T. Yoshida, R. Nakasima, J. Nucl. Sci. Technol. 18 (6), 393-407 (1981)

[25] A. Algora, et al., Phys. Rev. Lett. 105, 202501 (2010)

[26] J. Leppänen et al., Ann. Nucl. Energy 82, 142-150 (2015) 